\title{
USE OF DISTRIBUTION OF F-LATENCY (DFL) IN THE DETECTION OF CERVICAL SPONDYLOTIC NEUROPATHY
}

\author{
Md. Iftekhar Hossain ${ }^{1}$, Ehsan Alam Chowdhury, A.A Mamun', A Salam ${ }^{2}$ \\ Tanvir Noor Baig ${ }^{1}$ and K Siddique-e Rabbani
}

Department of Biomedical Physics \& Technology, University of Dhaka, Dhaka, Bangladesh email: ifti_ph@yahoo.com, ehsanac@live.com,rabbani@univdhaka.edu

\begin{abstract}
Distribution of F-latencies (DFL) is a new nerve conduction parameter introduced by our laboratory to give a distribution of the conduction velocity (DCV) of motor nerve fibres in a peripheral nerve trunk simply as its mirror image. Earlier work revealed an association between Cervical Spondylotic (CS) neuropathy and DFL patterns obtained from the thenar muscle at the base of the thumb by artificially stimulating the median nerve. DFL froom normal subjects had a single peaked pattern, while that from subjects with CS had double or triple peaks, or a broad peak. The broad peak was suggested to be an indicator of early CS, even when the symptoms are not fully apparent. This led to some hypotheses based on physics, physiology and statistics, in order to explain such patterns. In order to verify these hypotheses, experiments need to be carried out carefully, and the present work is a step towards that goal. In this work DFL of the median nerve was obtained from 15 subjects, 6 normal, and 9 with diagnosed cervical spondylosis. The latter were subjected to further X-ray and MRI investigations, performed blindly at regular clinics. All of the 9 subjects were identified with either compression of nerve roots (radiculopathy), or spinal cord compression due to the bulging of the inter-vertebral discs (myelopathy). This shows with confidence that double or triple peaks of DFL indeed relates to CS, and broad peaks indicate their early stage. The same concept may be extended to other peripheral nerves, both in the hands and legs. Thus DFL may become an important screening test for neuropathy, as a first choice, offering a low cost and widely available test compared to the only other alternative, MRI, for such diagnosis.
\end{abstract}

Keywords: Nerve conduction, Distribution of Conduction Velocity, Distribution of F-Latency, DCV, DFL, Cervical Spondylosis, Radiculopathy, Myelopathy.

\section{INTRODUCTION}

Earlier work in our laboratory established a new nerve conduction measurement parameter named Distribution of F-latency (DFL), obtained from evoked EMG responses, and established that it is an approximate mirror image of Distribution of Conduction Velocity (DCV) of motor nerve fibres in a nerve trunk, an important neuro-physiological parameter for diagnosis [1]. Before this work there was no practical method for obtaining DCV suitable in a clinical environment. It was also found in the initial experiments that DFL, obtained from the thenar muscle at the base of the thumb by stimulating the median nerve, has a single peak for normal nerves while for Cervical Spondylotic (CS) neuropathy, it shows double or triple peaks [2]. Besides, sometimes DFL has a broad peak, which has been suggested as an early stage of CS neuropathy that is still sub-clinical.

\footnotetext{
${ }^{1}$ Department of Physics, University of Dhaka, Dhaka, Bangladesh

${ }^{2}$ Bangladesh Institute for Biomedical Engineering \& Appropriate Technology (BIBEAT)
} 
To explain the occurrence of such multiple peaks or a broad peak, a number of hypotheses were put forward [3], and are summarized below. The present work is a step towards verification of these hypotheses.

The Thenar muscle (Abductor Policis Brevis, APB) at the base of the thumb is served by the median nerve with nerve branches $\mathrm{C} 7, \mathrm{C} 8$ and $\mathrm{T} 1$ at the vertebral levels. It was hypothesized in the above work that compression of any one of these branches at the spinal root will cause fibres through that nerve branch to contribute to a delayed DFL while fibres through the other two uncompressed nerve branches will contribute to a DFL at the original position. When DFLs due to all of these nerve branches are combined, a double peak will result. If two of the nerve branches are compressed to different degrees, and one remains uncompressed, triple peaks may result. This hypothesis tries to explain multiple peaks of DFL on the basis of nerve branch compression at the root, or, radiculopathy.

Double peaks of DFL were also observed in some subjects who did not have radiculopathy, but had bulging discs pressing onto the spinal cord at a higher inter-vertebral level, typically at C5-6 or C4-5. This condition is known as myelopathy and another hypothesis was put forward to explain this condition. It was suggested that the bulging disc pressed onto descending nerve fibres from the brain some of which ended up and linked to cell bodies of peripheral motor nerve branches coming out at immediately lower levels. Because of the pressure the descending nerves may get degenerated, which in turn would cause the linked peripheral nerves to degenerate. This would happen because the body has a tendency to shrink organs or tissues that are not in use (disuse atrophy). Therefore nerve fibres through one or more of the branches $\mathrm{C} 7, \mathrm{C} 8$ and $\mathrm{T} 1$ may be degenerated, with reduction in conduction velocity $(\mathrm{CV})$. Fibres with such reduced $\mathrm{CV}$ will contribute to a delay shifted DFL, and again, together with the non-degenerated fibres, will contribute to double peaks in the measured DFL.

Broad peaks were explained as the early stage of the above disorders, when the delay in the DFL segment is not enough to get resolved. Usually no clinical symptoms appeared for such observations and the broad peaks were suggested to be useful in providing early indications of neuropathy.

The above information suggest that DFL would become a very important and sensitive indicator of neuropathy, if the hypotheses presented could be proved through real life measurements. The present work, as mentioned above, was taken up with this aim.

\section{METHODS}

Firstly the study was performed on 6 volunteers reported to have diagnosed Cervical Spondylosis. Then another 6 subjects were chosen who did not have any neurological complain. These subjects consisted mainly of students of Dhaka University and their acquaintances. Some subjects were elderly relatives of these students and the age ranged from 25 to 64 years.

DFLs were obtained from both median nerves of each subject, using standard techniques developed by our group earlier and briefly mentioned below. Since the technique of measurement is nothing different from the routine measurements of nerve conduction for diagnosis which is non-invasive and does not pose any hazard, only oral consent was taken from the subjects.

To obtain DFL, 30 to 40 stimuli were applied to a median nerve at the wrist and the evoked EMG signals were obtained from the corresponding thenar muscle (abductor policies brevis, APB). The tests were performed at room temperature which was more or less stable at around $30^{\circ} \mathrm{C}$. F-latencies for the 
resulting F-responses were sorted into latency 'bins' of width $2 \mathrm{~ms}$. The number of occurrences in each bin was plotted against the latency mean and the resultant histogram smoothed to give the frequency distribution which is the DFL. Typically three patterns of DFL were observed as described in the earlier work and reported before, i.e., single peak, clearly defined double peak and broad peak.

Subsequently 3 more subjects were added into the study who had diagnosed CS as well. This made 9 subjects with diagnosed CS. X-ray and MRI investigations of the cervical spine was performed on some of these subjects. These investigations were performed at regular clinics where the investigator did not have any chance to know that this was part of a research. Not all had both the tests because of some logistic difficulties, however, useful and interesting information could be obtained to test the hypothesis mentioned above. One from the 6 normal subjects was also tested using X-ray investigation.

\section{RESULTS}

The results of the first study on the 12 subjects (6 normal and 6 with CS) are presented in Table 1. As expected, the 6 normal subjects demonstrated single peak of DFL on both sides, and they did not have any neurological complain either (serial 1 to 6 in Table 1). One of them was investigated using X-ray, and the report came out normal, with no bony growth.

Table 1: DFL of 12 subjects to relate their clinical condition

\begin{tabular}{|c|c|c|c|c|c|}
\hline \multirow{2}{*}{$\begin{array}{c}\text { Subject } \\
\text { No. }\end{array}$} & \multirow{2}{*}{$\begin{array}{c}\text { Subject } \\
\text { ID }\end{array}$} & \multirow{2}{*}{$\begin{array}{c}\text { Age, } \\
\text { Yrs }\end{array}$} & \multicolumn{2}{|c|}{ No. of DFL peak(s) } & \multirow{2}{*}{$\begin{array}{l}\text { Clinical } \\
\text { condition }\end{array}$} \\
\hline & & & $\begin{array}{c}\text { Right } \\
\text { median }\end{array}$ & $\begin{array}{c}\text { Left } \\
\text { median }\end{array}$ & \\
\hline 1 & BPL & 25 & S & $S$ & $\mathrm{NC}$ \\
\hline 2 & SHA & 25 & S & $S$ & $\mathrm{NC}$ \\
\hline 3 & ARA & 25 & S & S & $\mathrm{NC}$ \\
\hline 4 & SID & 26 & S & $S$ & $\mathrm{NC}$ \\
\hline 5 & TAZ & 26 & S & $\mathrm{S}$ & $\mathrm{NC}$ \\
\hline 6 & IFT & 26 & $\mathrm{~S}$ & $\mathrm{~S}$ & $\mathrm{NC}$ \\
\hline 7 & HFT & 26 & $\mathrm{D}$ & $S$ & $\mathrm{CS}$ \\
\hline 8 & $\mathrm{CHA}$ & 26 & $\mathrm{D}$ & $\mathrm{D}$ & $\mathrm{CS}$ \\
\hline 9 & EHS & 36 & $S$ & $\mathrm{D}$ & CS \\
\hline 10 & AKT & 46 & $\mathrm{~T}$ & $\mathrm{~T}$ & $\mathrm{CS}$ \\
\hline 11 & SAL & 49 & S & $\mathrm{D}$ & CS \\
\hline 12 & HUQ & 64 & $\mathrm{D}$ & $\mathrm{D}$ & $\mathrm{CS}$ \\
\hline \multicolumn{6}{|c|}{$\begin{array}{l}\text { Abbreviations } \\
\text { S: Single peak; D: Double peak; T: Triple peak; } \\
\text { CS: Diagnosed Cervical Spondylosis with clinical complain; } \\
\text { NC: No Clinical Complain; }\end{array}$} \\
\hline
\end{tabular}


All of the remaining 6 subjects (serial 7 to 12 in Table 1) having diagnosed Cervical Spondylosis together with clinical symptoms, demonstrated double or triple peaks of DFL. As mentioned above, 3 more subjects with diagnosed CS was added to this group later. The results of the X-ray and MRI investigations on these 9 subjects with diagnosed CS are presented in Table 2.

It is interesting to note that one of the subjects (KSR) had a broad peak with indication of two humps two years back, before the present work was taken up. His MRI at that time indicated mild disc compression on the spinal cord (myelopathy) at C5-6 intervertebral space. At present his DFL shows clear double peaks, and he feels a burning sensation around the neck and shoulders when the arms are kept high on the table, indicating mild cervical spondylotic neuropathy. This also shows that the broad peak of DFL two years back indicated the early stage when there were no symptoms.

Table 2: X-ray and MRI findings of subjects having double peak of DFL

\begin{tabular}{|l|c|l|l|}
\hline Subject ID & $\begin{array}{c}\text { Age, } \\
\text { yrs }\end{array}$ & X-ray findings (for whom done) & MRI findings (for whom done) \\
\hline HFT & 26 & Mild ost: C5, DSR & ---- \\
\hline CHA & 26 & Marginal ost: C4, DSR & --- \\
\hline EHS & 36 & Marginal ost C7, DSR & DB: C4-C5, C5-C6, SCC \\
\hline AKT & 46 & Mild ost: C5-C6, DSR at C4-5 and C5-6 & --- \\
\hline SAL & 49 & $\begin{array}{l}\text { Ost: C5, C6, C7. } \\
\text { DSR at C5-6 }\end{array}$ & DB: C5-C6, SCC \\
\hline HUQ & 64 & $\begin{array}{l}\text { Mild ost: C4, C5, } \\
\text { DSR at C3-4, C5-6, C6-7. }\end{array}$ & --- \\
\hline KSR & 60 & & DB: C5-C6, SCC \\
\hline ARA & 25 & Cervical Rib & DB: C4-5, C5-6, SCC \\
\hline AAM & 26 & Normal & $\begin{array}{l}\text { DB: Disc bulging } \\
\text { SCC: Spinal cord compression }\end{array}$ \\
\hline \multicolumn{2}{|c|}{ Abbreviations: } & $\begin{array}{l}\text { Ost: Osteophyte } \\
\text { DSR: Disc space reduction }\end{array}$ \\
\hline
\end{tabular}

\section{DISCUSSIONS}

Tables 1 and 2 indicate very conclusively that all subjects having diagnosed CS had double or triple peaks of DFL, in at least one side, while all in the normal group had single peaks. Again from Table 2, it can be seen that X-ray and MRI investigation carried out during this work also indicates some sort of spinal abnormality affecting the nerves in all the 9 subjects who demonstrated double or triple peaks of DFL.

If radiculopathy has to affect the thenar muscle, there should be compression of $\mathrm{C} 7, \mathrm{C} 8$ or $\mathrm{T} 1$ nerve branches. In Table 2 two subjects (EHS and SAL) had bony growth (osteophyte) at C7, as revealed by Xray investigation, which may compress the nerve branch $\mathrm{C}$, and cause radiculopathy. There is also a case of cervical rib. However, in all others, disk space reduction coupled with disc bulging and compression on spinal cord are common at slightly higher levels of the vertebra. Therefore, it can be said that these cases had myelopathy, i.e., pressure on the spinal cord. This small study also indicates that myelopathy has affected more of the subjects than radiculopathy. 
The application of DFL in cervical spondylotic neuropathy came up almost unexpectedly, while the first attempts to establish DFL was being carried out by workers in our laboratory earlier. Details of X-ray or MRI investigations of that study were not available, but a retrospective analysis of the results was very indicative of an association. The hypotheses put forward to explain the above findings based on physiology, physics and statistics paved the way for taking up further experiments. The present work is a step towards such a methodical study incorporating X-ray and MRI investigations, carried out blindly. The findings support the above association and establishes the efficacy of DFL in the screening of cervical spondylotic neuropathy on a stronger footing.

Of course, such multiple peaks of DFL may occur because of various other causes as well, such as tumour, inflammation and nerve injury. Therefore, DFL may be an initial screening method for any peripheral neurological disorder, a detailed one taken up later for positive cases, thus saving a great deal on health care. Once an equipment is established there is virtually no material cost in obtaining investigations on nerve conduction including DFL. Besides, equipment can be made portable and low cost if these are made locally, and could be made available widely. Therefore, DFL has all the merits of being a routine screening test for neuropathy.

We carry out regular clinical work where DFL is routinely performed on the appropriate peripheral nerves. This is being carried out over the last 6 years and has resulted in improved diagnosis. Our understanding is that nerve trunk compression at any point distal to the junction of the nerve branches will lead to an overall delay shift of DFL as for CTS, while compression or degeneration of one or more of the few nerve branches near the roots will lead to multiple peaks of DFL. We have also noticed double peaks of DFL for subjects with external injuries affecting the peripheral nerves slightly. This needs a rigorous study again. However, the observations are very reliable and reproducible enough to be taken into cognisance. Double peaks were also observed from Tibial and C. Peroneal nerves for subjects with Lumbo-sacral (LS) spondylotic neuropathy. Combining all these findings we may say that double or triple peaks of DFL definitely indicate some sort of neuropathy, most possibly of spinal origin, if no history of injury is present. Any entrapment of the nerve trunk after the spinal nerve branches have combined will lead to an overall delay shift of DFL which can be identified through a comparison with that from the other side, since such disorders are not exactly equal on both sides. Therefore, our work establishes that the presence of a broad peak or more than one peak in DFL of the peripheral nerves may be taken as an indicator of neuropathy. This can become a first investigation of choice in any peripheral neuropathy, which may include CS and LS Spondylotic neuropathy, as well as inflammation, tumour, etc. Further work may lead to differential diagnosis as well.

\section{CONCLUSIONS}

DFL appears to have brought in a new dimension in nerve conduction measurements, and has the potential to become a standard method of choice in the investigation of peripheral neuropathy. DFL gives an inner view into the nerve trunk using a simple measurement methodology, which no other existing clinically suitable method provides. Of course it is limited to motor fibres only, and to those that take part in F-responses, still it does have a great significance and importance in neural diagnosis. Many neurological disorders relate to motor nerves first. DFL may provide useful diagnostic information for motor nerves, which could then be used to assess neuropathy in sensory nerves through expertise and experience of the clinician. Together with conventional nerve conduction techniques, DFL has the potential of providing an improved diagnosis, with confidence. 


\section{ACKNOWLEDGEMENTS}

The authors wish to acknowledge Professor A F M Ruhal Haque for providing space and other logistic support at Trauma Centre, Shyamoli, Dhaka, where the equipment was set up and the studies undertaken. The authors also wish to acknowledge the generous financial support by Mr. Sheikh Jamiluddin, Director of Akij Food and Dairy Products Ltd., to carry out the X-ray and MRI investigations used in this study.

\section{REFERENCES}

1. Rabbani KS, Alam MJ, Salam MA. Frequency Distribution of F-Latencies (DFL) has physiological significance and gives Distribution of Conduction Velocity (DCV) of motor nerve fibres with implications for diagnosis. J of Biol Phys. (Springer), 2007;33:291-303.

2. Alam MJ, Rabbani KS, Possible detection of cervical spondylotic neuropathy using Distribution of F-latency (DFL), a new neurophysiological parameter, BMC Research Notes, 3:112, 2010, <http://www.biomedcentral.com/1756-0500/3/112>.

3. Rabbani, KS, Explaining the Occurrence of Multiple Peaks of DFL in Nerve Conduction Measurement, Bangladesh Journal of Medical Physics, Vol.4, January 2011 (Current issue of this journal) 